\title{
Comparison of contrast-enhanced CT with diffusion -weighted MRI in the Evaluation of patients with acute biliary pancreatitis
}

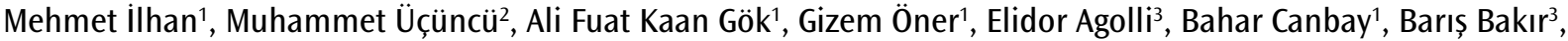 \\ Recep Güloğlu' ${ }^{1}$, Cemalettin Ertekin ${ }^{1}$
}

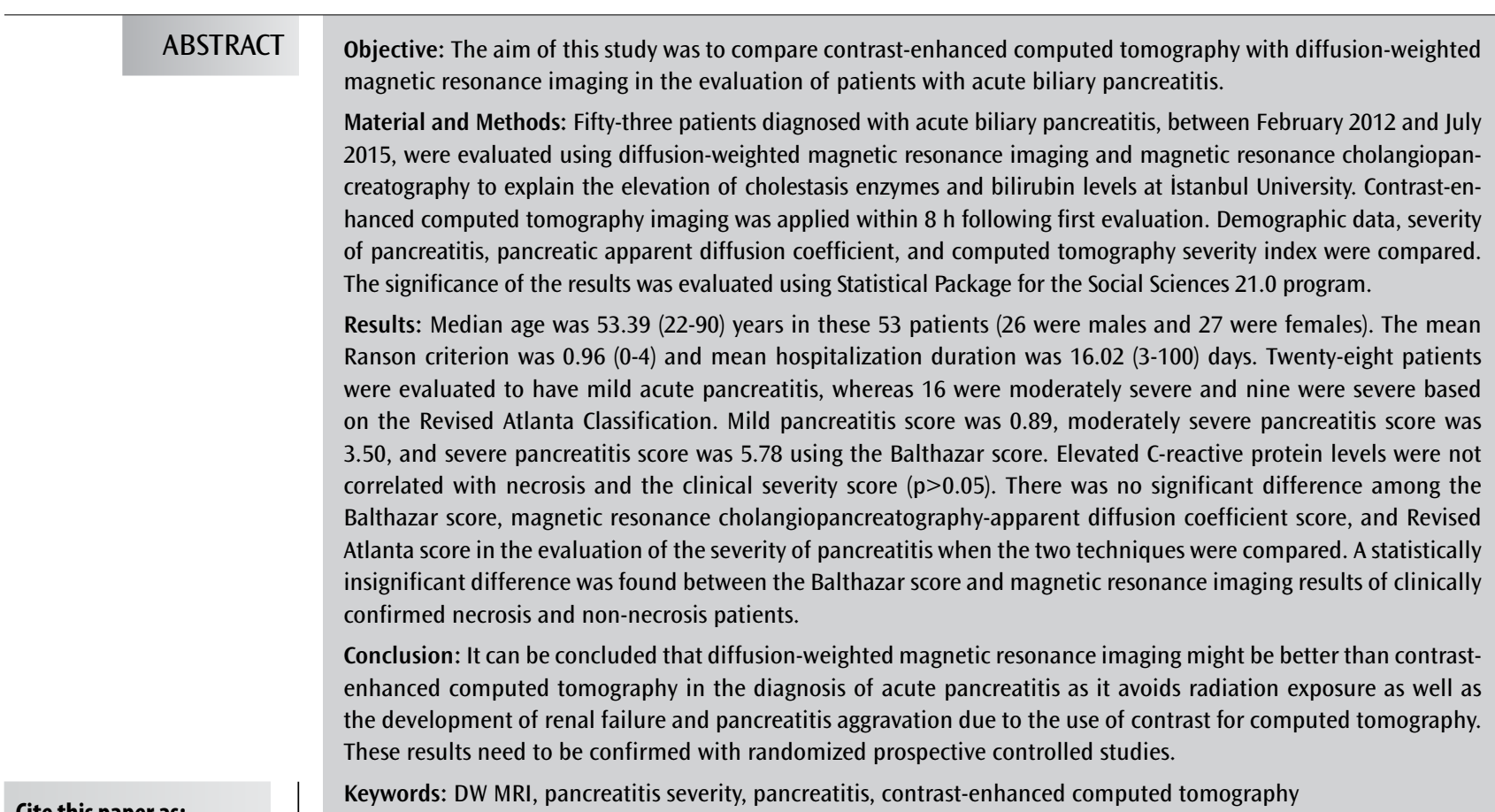

Cite this paper as:

İlhan M, Üçüncü M, Gök AFK, Öner Ö, Agolli E, Canbay C, Bakır B, Güloğlu R, Ertekin C. Comparison of contrastenhanced CT with diffusion -weighted MRI in the Evaluation of patients with acute biliary pancreatitis. Turk J Surg 2017; 33: 153-157.

'Department of General Surgery, İstanbul University Istanbul School of Medicine, İstanbul, Turkey ${ }^{2} C$ linic of General Surgery, Arnavutköy State Hospital, İstanbul, Turkey

${ }^{3}$ Department of Radiology, Istanbul University İstanbul School of

Medicine, İstanbul, Turkey

Address for Correspondence Gizem Öner

e-mail:

gizem.oner@istanbul.edu.tr

Received: 04.01.2016

Accepted: 28.05.2016

CCopyright 2017

by Turkish Surgical Association

Available online at

www.turkjsurg.com

\section{INTRODUCTION}

Acute pancreatitis progresses on an instable course that has exacerbations and remissions. The mortality rate is between $2.1 \%$ and $7.8 \%$. Development of necrosis increases mortality in pancreatitis. In patients with necrosis, the mortality rate increases up to $25 \%(1,2)$. Contrast-enhanced computed tomography $(\mathrm{CT})$ is the most important imaging technique to determine the severity of pancreatitis. However, recent studies suggest that the contrast agent for $\mathrm{CT}$ aggravates pancreatitis and provokes organ failure. In addition, the inability to administer contrast to patients with renal dysfunction and contrast allergy causes the disease to be assessed insufficiently (3).

Limitations of CT have canalized clinicians to consider different imaging studies. It is believed that diffusion-weighted magnetic resonance imaging (DW MRI) may be compared to and may even replace CT. The severity of acute biliary pancreatitis was evaluated with contrast-enhanced CT and it was compared with DW MRI.

\section{MATERIAL AND METHODS}

\section{Patients}

The patients diagnosed with acute biliary pancreatitis in the Department of General Surgery of istanbul University by the School of Medicine using DW MRI and MRCP when cholestasis enzyme levels or bilirubin levels were elevated at the time of initial diagnosis were taken for a CT scan within $8 \mathrm{~h}$. The results of two imaging techniques were compared. None of the patients had imaging contraindications such as metallic implant or claustrophobia for DW MRI. The patients' questionnaire included history of hepatic or biliary operations, hepatotoxic drug use, chronic alcohol use, hepatitis B or C carrier status, and suspicion of periam- 
pullary tumor. The patients were excluded from the study in the presence of above conditions. Informed consent was obtained from all participants, and the study followed the guidelines of the Declaration of Helsinki. As this study was retrospectively performed by scanning patient files and imaging methods, it was exempt from institutional ethics committee approval.

The diagnosis of acute biliary pancreatitis was confirmed with increased serum and urine amylase levels. The biochemical parameters such as aspartate transaminase (AST), alanine aminotransferase (ALT), alkaline phosphatase (ALP), gammaglutamyl transferase (GGT), lactate dehydrogenase (LDH), total bilirubin (TBIL), and direct bilirubin (DBIL) were evaluated at the initial time of admission to the hospital. Ranson value and Balthazar score were also assessed at the initial time of diagnosis and within $24 \mathrm{~h}$. C-reactive protein (CRP) levels at the time of first admission and the highest levels of CRP were recorded.

The cases were classified as having mild, moderate, and severe pancreatitis according to the Revised Atlanta classification. CT scan was performed when the general condition worsened and acute-phase reactants increased. Ultrasound- or CT-guided percutaneous drainage was performed when there was a necessity to drain the collection according to results of imaging techniques. Endoscopic retroperitoneal drainage was also performed when percutaneous drainage was evaluated as insufficient. Cholecystectomy was performed in patients when the pancreatitis regressed prior to discharge.

\section{CT Protocol}

Computed tomography was performed in supine position with hands over head, with intravenous contrast using a 16 detector PHILIPS device. The images were taken in the $60^{\text {th }}$ second after the administration of the intravenous contrast. CT severity score was assessed (Table 1) (4).

Diffusion-Weighted Magnetic Resonance Imaging Protocol Gyroscan Intera Master (1.5 T; Philips Medical Systems, Best, The Netherlands) was used for MRI. DW MRI was performed in the axial plane with a spin-echo echo-planar imaging, singleshot sequence [repetition time (RT) $3505 \mathrm{~ms}$, echo time (ET) $68 \mathrm{~ms}$, fl ip angle, $\left.90^{\circ}\right]$, and b values of 0 and $1000 \mathrm{~s} / \mathrm{mm}^{2}$ with a four-channel sense body coil. A respiratory trigger was not used; the scan was performed under free-breathing conditions. Fifty slices were produced with a 7-mm slice thickness and a 1-mm interslice gap. Other parameters were field of view (FOV), 375 mm; matrix, 124 X100; and double number of samples averaged (NSA) sense factor, 3.0. An apparent diffusion coefficient (ADC) map was obtained for each slice position (5-7).

\section{Statistical Analysis}

The results of the evaluation techniques and patient characteristics were compared using statistical methods. The findings were evaluated by IBM Statistical Package for the Social Sciences 21 (IBM Corp.; Armonk, NY, USA). Data was presented as median, minimum, maximum, standard deviation, and mean. The distribution of the variables was analyzed with the Shapiro-Wilk test. In comparison between the two groups, the Mann-Whitney $U$ test was used. Kruskal-Wallis test was used for comparison of more than two groups. Variables of the pa-
Table 1. CT severity score in patients with pancreatitis

Pancreatitis staging in imaging without contrast

Point (A)

Normal pancreas

0

Pancreatic expansion

1

Inflammation of pancreatic or peripancreatic fatty tissue $\quad 2$

One peripancreatic fluid collection

3

Two or more fluid collections or Retroperitoneal air

4

$\begin{array}{lc}\text { Pancreatitis staging in contrast enhanced imaging } & \text { Point (B) } \\ \text { There is not pancreatic necrosis } & 0 \\ 30 \%>\text { pancreatic necrosis } & 2 \\ 50 \%>\ldots>\% 30 \text { pancreatic necrosis } & 4 \\ 50 \&<\text { pancreatic necrosis } & 6 \\ \text { CT severity index (CTSI) } & \text { A+B } \\ \text { Mild pancreatitis } & 0-2 \\ \text { Moderate pancreatitis } & 3-6 \\ \text { Severe pancreatitis } & 7-10\end{array}$

Table 2. Distribution of patients' biochemical parameters at the time of admission

\begin{tabular}{|lccccc|}
\hline N=53 & Mean & $\begin{array}{c}\text { Standard } \\
\text { deviation }\end{array}$ & Median & Minimum & Maximum \\
\hline WBC & 12714 & 4571 & 12100 & 4900 & 26200 \\
\hline ALT & 193 & 219 & 70 & 11 & 870 \\
\hline ALP & 181 & 162 & 117 & 49 & 709 \\
\hline GGT & 274 & 256 & 205 & 10 & 1092 \\
\hline LDH & 613 & 235 & 524 & 256 & 1135 \\
\hline TBIL & 1.9 & 2.0 & 1.0 & 0.30 & 7.7 \\
\hline DBIL & 1.3 & 1.7 & .4 & 0.01 & 6.8 \\
\hline CRP & 78 & 126 & 11 & 0 & 436 \\
\hline
\end{tabular}

WBC: white blood cell count; ALT: alanine aminotransferase aspartate; ALP: alkaline phosphatase; GGT: gamma-glutamyl transferase; LDH: lactate dehydrogenase; TBIL: total bilirubin; DBIL: direct bilirubin; CRP: C-reactive protein

by receiver operator characteristic (ROC) analysis. The results were in the $95 \%$ confidence interval and the significance was assessed at the level of $p<0.05$.

\section{RESULTS}

A total of 53 cases ( 26 males and 27 females) were included in the study; mean age was 55.39 (22-90) years. The median and range values of biochemical parameters, such as AST, ALT, ALP, GGT, LDH, TBIL, and DBIL, of the cases at the time of admission are presented in Table 2.

In our study, mean Ranson values were determined as 1.10 (04). The median and range of CRP values of the cases at the time of presentation and when they were the highest were 78.06 (0.2-436) and 243.16 (3.7-640), respectively. Elevated CRP levels and presence of necrosis were not associated with clinical severity. According to the Revised Atlanta Score, 28 cases were mild, 16 cases were moderate, and 9 cases were se- 
Table 3. Comparison of clinical severity according to Ranson Score, Apparent diffusion coefficient (ADC), and Balthazar scores

\begin{tabular}{|c|c|c|c|c|c|c|c|c|c|}
\hline & \multicolumn{3}{|c|}{ Mild } & \multicolumn{3}{|c|}{ Moderate } & \multicolumn{3}{|c|}{ Severe } \\
\hline & Mean & $\begin{array}{l}\text { Standard } \\
\text { deviation }\end{array}$ & Median & Mean & $\begin{array}{l}\text { Standard } \\
\text { deviation }\end{array}$ & Median & Mean & $\begin{array}{l}\text { Standard } \\
\text { deviation }\end{array}$ & Median \\
\hline Ranson & 0.68 & 0.77 & 0.5 & 1.31 & 1.01 & 1 & 1.22 & 0.97 & 1 \\
\hline Balthazar score & 0.89 & 0.99 & 0.5 & 3.5 & 2.28 & 3 & 5.78 & 2.73 & 5 \\
\hline ADC & 1.33 & 0.15 & 1.32 & 1.38 & 0.4 & 1.32 & 1.57 & 0.35 & 1.7 \\
\hline
\end{tabular}

Table 4. Apparent diffusion coefficient (ADC) measurements and necrosis frequency according to pancreatitis severity

\begin{tabular}{|lcccc} 
& & Mild (\%) & Moderate (\%) & Severe (\%) \\
\hline Necrosis & - & $28.0(100)$ & $13.0(81)$ & $1.0(11)$ \\
& + & $0.0(0)$ & $3.0(19)$ & $8.0(89)$ \\
\hline ADC & $1.33 \pm 0.15$ & $1.38 \pm 0.40$ & $1.57 \pm 0.35$ & \\
\hline
\end{tabular}

ADC: apparent diffusion coefficient

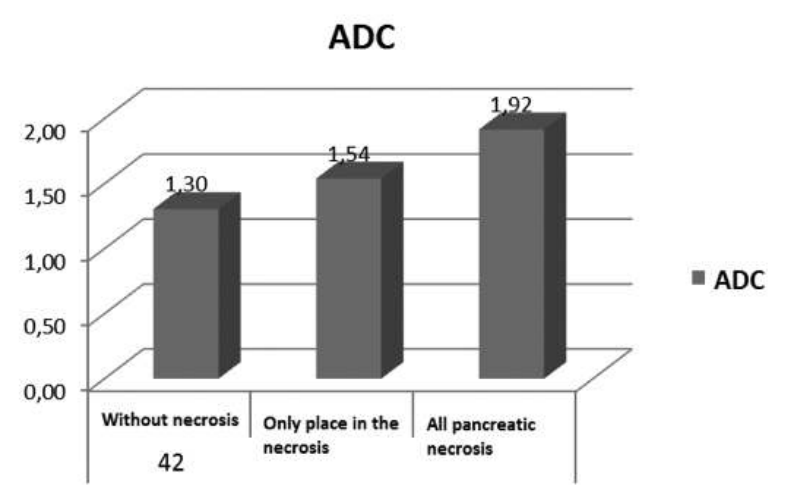

Figure 1. Comprasion based on necrosis location

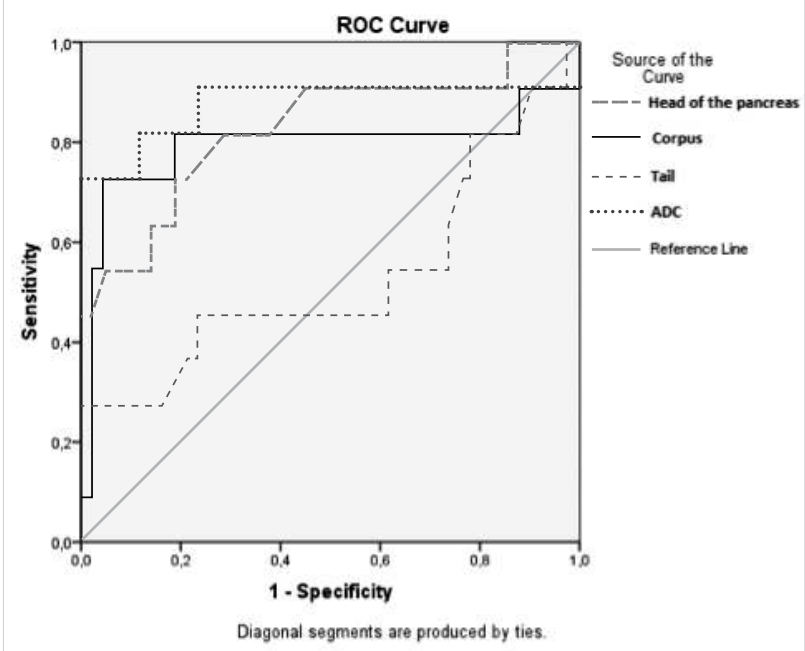

Figure 2. ROC analysis based on necrosis location

vere. Necrosis was observed in all severe cases and in two of the moderate cases. The Balthazar score of the mild, moderate, and severe cases was $0.7,3.16,5.37$, respectively.

There was no significant difference among the Balthazar score, MR ADC score and Revised Atlanta Score with regard to the evaluation of the severity of pancreatitis when the two techniques were compared. A statistically insignificant difference was found between the Balthazar score and MRI results of clinically confirmed necrosis and non-necrosis patients (Table 3).

Necrosis was not detected in 28 patients who had mild pancreatitis. Necrosis was detected in three of the moderate pancreatitis patients. Necrosis was not detected in one patient who had severe pancreatitis (Table 4).

There was a statistically significant difference in ADC measurements between the patients who had necrosis and those who did not $(p<0.001)$. There was also a statistically significant difference between the patients who had a single necrosis zone and those who had multiple necrosis zones (Figure 1, 2).

Four cases were monitored in the intensive care unit. Mean hospital stay was 16.02 days. Laparoscopic cholecystectomy was performed before discharge in 30 cases whose pancreatitis regressed. Percutaneous discharge was performed in two cases. In two of these cases, endoscopic retroperitoneal necrotic debridement was performed because of insufficient drainage. Seventeen cases were discharged after recovery and an elective cholecystectomy was planned. MRI and CT images of two patients are shown in Figure 3, 4.

\section{DISCUSSION}

Acute pancreatitis, which is the inflammation of pancreas, can present in a large spectrum, from self-limiting disease to a serious clinical presentation that can lead to sepsis and death. The systemic inflammatory response and complications accompanying pancreatitis cause an increase in disease-related mortality rates (8-10). Despite technological advances, it is debatable to distinguish the patients at increased risk for severe disease at the time of admission to the hospital. For scoring, Ranson criteria, Acute Physiologic and Chronic Health Evaluation (APACHE) II criteria, Balthazar score, and Bedside Index of Severity in Acute Pancreatitis (BISAP) are used (11-14).

In the Revised Atlanta classification, pancreatitis is classified as mild, moderate, and severe. While mortality in mild pancreatitis is $<1 \%$, mortality can rise up to $10 \%$ in cases with sterile necrosis and up to $30 \%$ in cases with severe necrosis $(8,15)$. The cases in our study were evaluated according to the Ranson criteria, Revised Atlanta classification, ADC values, and Balthazar score. According to Revised Atlanta Scoring, 28 of our cases were evaluated as mild, 16 of our cases were evaluated as moderate, and 9 of our cases were evaluated as severe. There was a difference between the Revised Atlanta Score and Balthazar score in terms of MRADC measurements, 

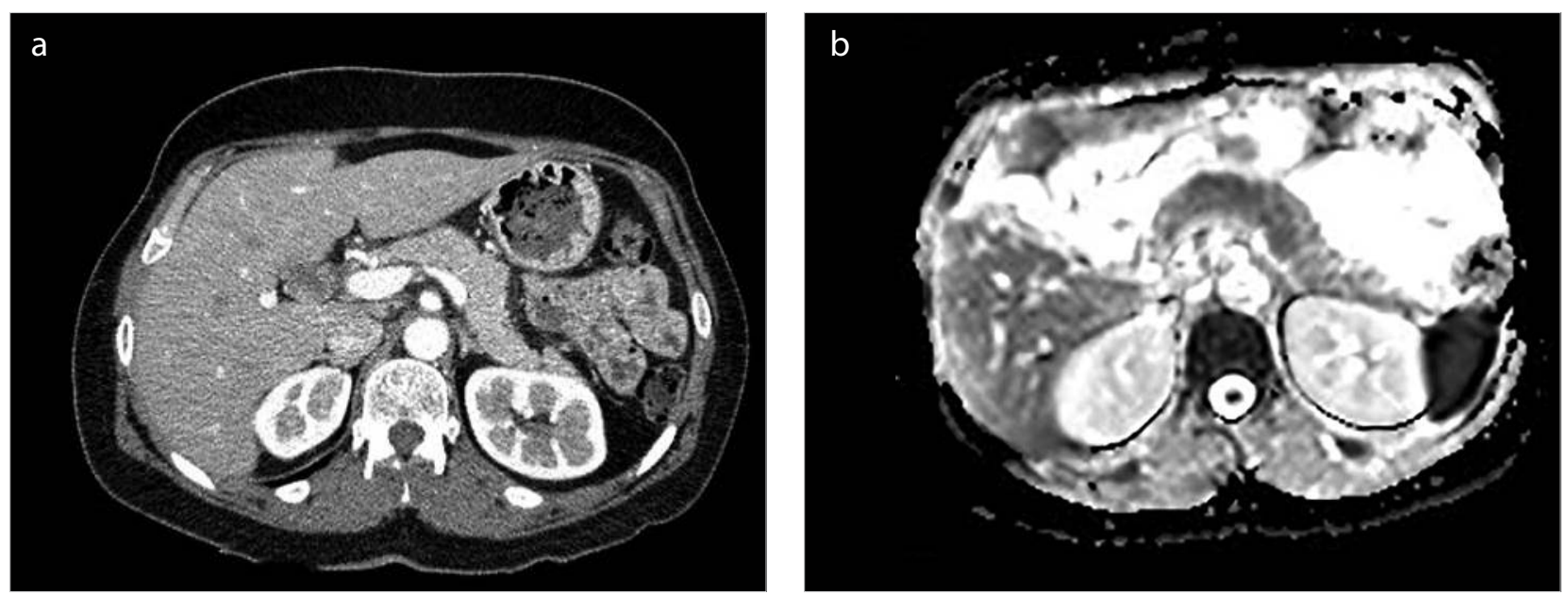

Figure 3. a, b. CT (a) and MRI (b) images of a 40-year-old male patient with necrosis
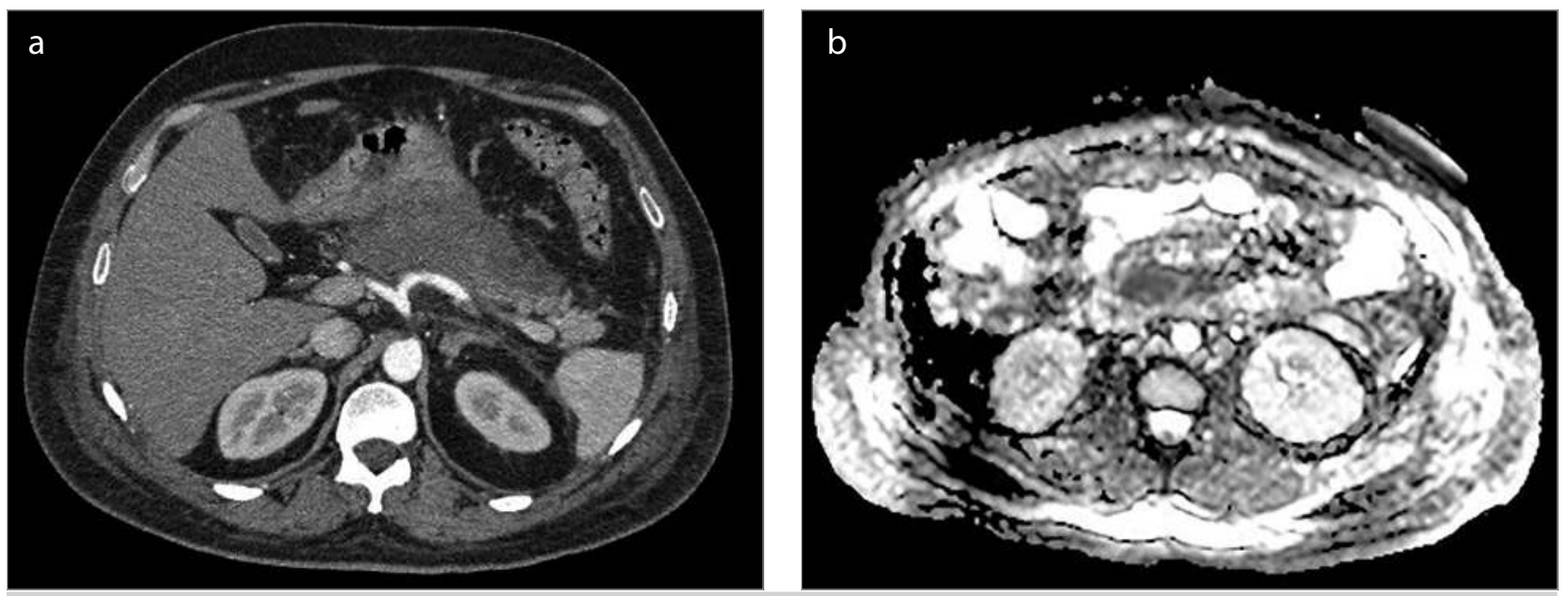

Figure 4. a, b. (a) CT and MRI (b) images of a 48-year-old female patient with acute pancreatitis

which was not statically significant. The mean Ranson value of our cases was determined as $1.10(0-4)$.

Intravenous contrast-enhanced $\mathrm{CT}$ is an imaging option that can be used for the diagnosis and determination of the severity of the disease. It is important to use CT for early diagnosis and grading of pancreatitis-related complications. CT at the time of first admission is not always necessary, and 15\%-30\% of CT imagings are non-pathological. The real contraindications for CT are renal insufficiency and contrast allergy. It is emphasized that intravenous contrast can impair pancreatic microcirculation, increase necrosis, and worsen pancreatitis. Despite these factors, CT is necessary to discover other acute abdominal problems that are not caused by pancreatitis.

The mortality rate is $15 \%$ in necrotizing pancreatitis. Infected necrosis is seen among one-third of cases with necrotizing pancreatitis. The mortality rate increases in infected necrosis compared with sterile necrosis $(16,17)$. Infected necrosis can be diagnosed by culture of needle aspiration and presence of air within necrosis can be interpreted as infection.

In order to evaluate acute and chronic inflammation of the pancreas parenchyma, the utilization of DW MRI has recently changes observed in acute pancreatitis. Shinya et al. (18) have reported DW MRI signal intensity changes in acute pancreatitis for the first time. However, their study has not demonstrated a measurable diffusion contrast appearance. In standard T2-weighted imaging, ADC measurement is necessary to differentiate from flare phenomenon $(18,19)$.

In the study performed by Thomas et al. (19), increased signal activity and decreased ADC measurements have been detected in patients with acute pancreatitis compared with the patients with a normal pancreatic tissue. In this study, ADC values were measured under $1.62 \times 10-3 \mathrm{~mm}^{2}$ that were evaluated as pancreatitis. When serum leukocyte, amylase, and CRP levels turned back to normal, control imaging was again performed to compare ADC values, which also turned back to normal (19). In our study, ADC measurement was under $1.62 \times 10-3 \mathrm{~mm}^{2}$ in all patients.

The limitations of our study include its retrospective nature, the paucity of cases, especially those that have necrosis, the fact that DW MRI was not performed when the patient clinically deteriorated, and so that the comparison of necrotic on the CT with DW MRI was also not performed. DW MRI of the patients is obtained either at the time of first admission or within the first week of admission. However, pancreatic necro- 
sis generally emerges three to four weeks after inflammation, which demonstrates the necessity for randomized prospective controlled studies.

\section{CONCLUSION}

Diffusion-weighted magnetic resonance imaging was superior to CT scan in the evaluation of cases with suspected pancreatitis as there is no radiation and contrast existence that protects the pancreas from exacerbation of pancreatitis. Therefore, DW MRI can be selected in the diagnosis of pancreatitis, especially in patients with organ deficiency, and in differential diagnosis of necrosis, and to decrease the complications of pancreatitis. Prospective randomized studies are needed for defining the definitive role of DW MRI in the evaluation of acute pancreatitis.

Ethics Committee Approval: Authors declared that the research was conducted according to the principles of the World Medical Association Declaration of Helsinki "Ethical Principles for Medical Research Involving Human Subjects" (amended in October 2013).

Informed Consent: Written informed consent was obtained from patients who participated in this study.

Peer-review: Externally peer-reviewed.

Author Contributions: Concept - M.İ, M.Ü., G.Ö.; Design - M.I.., G.Ö.; Supervision - C.E., E.A., R.G.; Resource - M.Ü., A.F.K.G.; Materials - G.Ö., E.A.; Data Collection and/or Processing - E.A., B.C.; Analysis and/or Interpretation - M. I., M.Ü., A.F.K.G.; Literature Search - G.Ö., B.B.; Writing Manuscript - M.I, M.Ü., G.Ö.; Critical Reviews - B.B., R.G., C.E.

Conflict of Interest: No conflict of interest was declared by the authors.

Financial Disclosure: The authors declared that this study has received no financial support.

\section{REFERENCES}

1. Huang W, Altaf $K$, Jin T, Xiong J-J, Wen L, Javed MA, et al. Prediction of the severity of acute pancreatitis on admission by urinary trypsinogen activation peptide: A meta-analysis World J Gastroenterol 2013; 19: 4607-4615. [CrossRef]

2. Senthil Kumar $\mathrm{P}$, Ravichandravn $\mathrm{P}$, Jeswanth $\mathrm{S}$. Case matched comparison study of the necrosectomy by retroperitoneal approach with transperitoneal approach for necrotizing pancreatitis in patients with CT severity score of 7 and above. Int J Surg 2012; 10: 587-592. [CrossRef]

3. Carmona-Sanchez R, Uscanga L, Bezaury-Rivas P, Robles-Diaz G, Suazo-Barahona J, Vargas-Vorackova F. Potential harmful effect of iodinated intravenous contrast medium on the clinical course of mild acute pancreatitis. Arch Surg 2000; 135: 1280-1284. [CrossRef]

4. Banks PA, Bollen TL, Dervenis C, Gooszen HG, Johnson CD, Sarr $M G$, et al. Classification of acute pancreatitis-2012: revision of the Atlanta classification and definitions by international consensus. Gut. 2013; 62: 102-111. [CrossRef]
5. Akisik MF, Aisen AM, Sandrasegaran K, Jennings SG, Lin C, Sherman $S$, et al. Assessment of chronic pancreatitis: Utility of diffusion weighted MR imaging with secretin enhancement. Radiology 2009; 250: 103-109. [CrossRef]

6. Taniguchi T, Kobayashi $H$, Nishikawa $K$, lida $E$, Michigami $Y$, Morimoto $\mathrm{E}$, et al. Diffusion-weighted magnetic resonance imaging in autoimmune pancreatitis. Jpn J Radiol 2009; 27: 138-142. [CrossRef]

7. Burdette JH, Elster AD, Ricci PE. Acute cerebral infarction: quantification of spin-density and T2 shine-through phenomena on diffusion-weighted MR images. Radiology 1999; 212: 333-339. [CrossRef]

8. Logue JA, Carter CR. Minimally invasive necrosectomy techniques in severe acute pancreatitis: role of percutaneous necrosectomy and video-assisted retroperitoneal debridement. Gastroenterol Res Pract 2015; 2015: 693040.

9. Larvin M, McMahon MJ. APACHE-II score for assessment and monitoring of acute pancreatitis. Lancet 1989; 2: 201-205. [CrossRef]

10. Petrov MS. Meta-analyses on the prophylactic use of antibiotics in acute pancreatitis: many are called but few are chosen. Am J Gastroenterol 2008; 103: 1837-1838. [CrossRef]

11. Wu BU, Johannes RS, Sun X, Tabak Y, Conwell DL, Banks PA. The early prediction of mortality in acute pancreatitis: a large population-based study. Gut 2008; 57: 1698-1703. [CrossRef]

12. Adam F, Bor C, Uyar M, Demırag K, Çankayalı I. Severe acute pancreatitis admitted to intensive care unit: SOFA is superior to Ranson's criteria and APACHE II in determining prognosis. Turk J Gastroenterol 2013; 24: 430-435. [CrossRef]

13. Yang YX, Li L. Evaluating the Ability of the Bedside Index for Severity of Acute Pancreatitis Score to Predict Severe Acute Pancreatitis: A Meta-Analysis. Med Princ Pract 2016; 25: 137-142. [CrossRef]

14. Banday IA, Gattoo I, Khan AM, Javeed J, Gupta G, Latief M. Modified Computed Tomography Severity Index for Evaluation of Acute Pancreatitis and its Correlation with Clinical Outcome: A Tertiary Care Hospital Based Observational Study. J Clin Diagn Res 2015; 9: TC01-5. [CrossRef]

15. Banks PA, Bollen TL, Dervenis C, Gooszen HG, Johnson CD, Sarr MG, et al. Classification of acute pancreatitis-2012: revision of the Atlanta classification and definitions by international consensus. Gut. 2013; 62: 102-111. [CrossRef]

16. Trikudanathan G, Attam R, Arain MA, Mallery S, Freeman M.L. Endoscopic interventions for necrotizing pancreatitis. Am. J. Gastroenterol. 2014; 109: 969-981. [CrossRef]

17. Lim E, Sundaraamoorthy RS, Tan D, Teh HS, Tan T-J, Cheng A. Stepup approach and video assisted retroperitoneal debridement in infected necrotizing pancreatitis: A case complicated by retroperitoneal bleeding and colonic fistula. Ann Med Surg (Lond) 2015; 4: 225-229. [CrossRef]

18. Shinya S, Sasaki T, Nakagawa Y, Guiquing Z, Yamamoto F, Yamashita $Y$. The efficacy of diffusion-weighted imaging for the detection and evaluation of acute pancreatitis. Hepatogastroenterology 2009; 56: 1407-1410.

19. Thomas S, Kayhan A, Lakadamyali H, Oto A. Diffusion MRI of acute pancreatitis and comparison with normal individuals using ADC values. Emerg Radiol 2012; 19: 5-9. [CrossRef] 